\title{
A new model to produce sagittal plane rotational induced diffuse axonal injuries
}

\author{
Johan Davidsson ${ }^{1}$ and Marten Risling ${ }^{2}$. \\ ${ }^{1}$ Vehicle Safety Division, Department of Applied Mechanics, Chalmers University of Technology, Göteborg, Sweden \\ 2 Experimental Traumatology, Department of Neuroscience, Karolinska Institutet, Stockholm, Sweden
}

\section{Edited by:}

Mattias Sköld, Uppsala University,

Sweden

Reviewed by:

Niklas Marklund, University of Uppsala, Sweden

Bridgette D. Semple, University of

California San Francisco, USA

*Correspondence:

Marten Risling, Department of

Neuroscience, Karolinska Institutet,

Retzius väg 8, 17177 Stockholm,

Sweden.

e-mail: marten.risling@ki.se
A new in vivo animal model that produces diffuse brain injuries in sagittal plane rearward rotational acceleration has been developed. In this model, the skull of an anesthetized adult rat is tightly secured to a rotating bar. During trauma, the bar is impacted by a striker that causes the bar and the animal head to rotate rearward; the acceleration phase last $0.4 \mathrm{~ms}$ and is followed by a rotation at constant speed and a gentle deceleration when the bar makes contact with a padded stop. The total head angle change is less than $30^{\circ}$. By adjusting the air pressure in the rifle used to accelerate the striker, resulting rotational acceleration between 0.3 and $2.1 \mathrm{Mrad} / \mathrm{s}^{2}$ can be produced. Numerous combinations of trauma levels, post-trauma survival times, brain and serum retrieval, and tissue preparation techniques were adopted to characterize this new model. The trauma caused subdural bleedings in animals exposed to severe trauma. Staining brain tissue with $\beta$-Amyloid Precursor Protein antibodies and FD Neurosilver that detect degenerating axons revealed wide spread axonal injuries (AI) in the corpus callosum, the border between the corpus callosum and cortex and in tracts in the brain stem. The observed Als were apparent only when the rotational acceleration level was moderate and above. On the contrary, only limited signs of contusion injuries were observed following trauma. Macrophage invasions, glial fibrillary acidic protein redistribution or hypertrophy, and blood brain barrier (BBB) changes were unusual. S100 serum analyses indicate that blood vessel and glia cell injuries occur following moderate levels of trauma despite the absence of obvious BBB injuries. We conclude that this rotational trauma model is capable of producing graded axonal injury, is repeatable and produces limited other types of traumatic brain injuries and as such is useful in the study of injury biomechanics, diagnostics, and treatment strategies following diffuse axonal injury.

Keywords: brain, animal model, diffuse axonal injury

\section{INTRODUCTION}

Traumatic brain injuries (TBI) represent approximately $60 \%$ of all deaths in hospitals among children and young adults in the western world (Melvin et al., 1993). Among survivors these injuries are often irreversible, causing long term pain, and disability. Although TBI can be associated with skull fractures, it commonly occurs without fractures (Gennarelli and Thibault, 1982). About 40\% of all TBI patients admitted to hospitals are non-focal injuries (Wismans et al., 2000) and are usually referred to as distributed brain injuries (DBI).

At least four categories of DBI can be identified: diffuse axonal injury (DAI); diffuse hypoxic, anoxic, or ischemic injury; diffuse swelling; and diffuse vascular injury. DAI is the most common type of DBI and commonly results in unconsciousness or death (Gennarelli et al., 1982; Melvin et al., 1993). The DAI pathology, which is characterized by perturbations to the axoplasmic transport along the length of axons (Povlishock and Jenkins, 1995), is likely to cause axonal swelling or degeneration which can reduce the functionality or disconnect the axons from their existing networks (Povlishock, 1992). It has been reported that DAI commonly are localized in the subcortical white matter, gray-white matter interface, and corpus callosum (Gennarelli et al., 1982; Ommaya, 1984; Smith and Meaney, 2000) as well as at points of attachment, such as cranial nerves (Viano, 1997).

Distributed brain injuries is commonly a result of inertial induced loads; intracranial motions arise when the skull is accelerated and the brain mass, due to its inertia, lags behind or continues its motion relative to the skull. These inertia induced loads are most common in rapid head rotations (Holbourn, 1943) which often occur in fall accidents, traffic accidents, and military assaults. It has been hypothesized that these inertia induced loads produce strains in the brain tissue and that these strains cause neurological deficiencies (Strich, 1961; Adams et al., 1989; Margulies et al., 1990; Margulies and Thibault, 1992; Povlishock, 1992; Zhang et al., 2004). Meaney et al. (1993) used numerical simulations to reconstruct experiments with miniature pigs (Ross et al., 1994) to determine the DAI-tolerance level on tissue level. For grade 1 and grade 2 DAI a maximum strain of 0.1 and 0.25 , respectively, was suggested. Using animal models, it has been suggested that the severity of DBI correlates with the amplitude of the angular acceleration (Abel et al., 1978; Ono et al., 1980; Margulies and Thibault, 1992). The use of detailed mathematical models of the 
head and brain, however, has indicated that injury correlates with the resulting angular velocity (Kleiven, 2007). In addition, duration of the impact has been reported to affect the injury type; short duration impacts result in a larger extent of focal injury, while long duration impacts mainly result in DBI (Margulies and Thibault, 1992). It has also been shown, in experiments with monkeys, that the incidence and degree of DBI correlated, although indirectly, with the direction of the head acceleration: coronal plane angular acceleration was the direction that caused the longest lasting coma, while sagittal plane angular accelerations and oblique accelerations produced coma for a shorter period (Gennarelli et al., 1982).

An attractive approach to studying DBI pathology and its associated injury mechanism and threshold would be to reconstruct well documented accident cases in which the patient is slightly injured. The real life accidents are however commonly rather complex and the patients suffer from a multitude of injuries. Therefore anesthetized animals have been used in the past to study DBI and DAI.

Primates were extensively used in the past but currently smaller animals such as miniature swine, rabbits, and rats are used (Table 1). In a model described by Marmarou et al. (1994), a cap the size of a dime is cemented to the denuded bone on top of the rat skull. During trauma this plate is impacted by a drop weight which causes the head to accelerate linearly and rotate forward in the sagittal plane. The initial purpose with the model was not to assess the threshold for DAI in rotational head trauma but rather to study the pathology and treatment of TBI in general. For head rotations in the coronal plane, Xiao-Sheng et al. (2000) exposed rats to $2 \mathrm{~ms}$ long rotational accelerations, after which they observed axonal swelling and bulblike protrusions on the axons in the medulla oblongata, midbrain, and corpus callosum. In two other studies by Ellingson et al. (2005) and by Fijalkowski et al. (2007), rats were exposed to higher rotational accelerations than in the study by Xiao-Sheng et al. (2000); $368 \mathrm{krad} / \mathrm{s}^{2}$ ) for approximately $2 \mathrm{~ms}$ in the coronal plane. Despite the higher accelerations, the rats suffered from classical concussion injuries with minimal histological abnormalities. A series of publications have presented a model in which rabbits are exposed to rotational acceleration in the sagittal plane (Gutierrez et al., 2001; Runnerstam et al., 2001; Hamberger et al., 2003; Hansson et al., 2003; Krave et al., 2005, 2011). Due to the size of the rabbit brain as compared to the rat brain, the rabbit has become the preferred choice. However, the rabbit brain is rather elongated as compared to the human brain.

Table 1 | Studies which exposed animal brains to rotational acceleration to improve the understanding of pathologenesis, injury mechanisms, and suggest injury threshold.

\begin{tabular}{|c|c|c|c|c|}
\hline $\begin{array}{l}\text { Plane of } \\
\text { motion }\end{array}$ & Subject used & $\begin{array}{l}\text { Method to assess injury } \\
\text { and type of injury produced }\end{array}$ & Concluding result & Reference \\
\hline \multirow[t]{3}{*}{$\begin{array}{l}\text { Sagittal plane lin. } \\
\text { and rot. acc. }\end{array}$} & Rat & Histology etc. & Developed to study TBI & $\begin{array}{l}\text { Marmarou et al. (1994) and } \\
\text { Foda and Marmarou (1994) }\end{array}$ \\
\hline & Rabbit & $\begin{array}{l}\text { Astrogliosis in hippocampus } \\
\text { and cerebral cortex, } \\
\text { hemorrhages, focal bleeding, } \\
\text { reactive astrocytosis, and } \\
\text { axonal injury }\end{array}$ & $\begin{array}{l}\text { Development of test rig, and } \\
\text { rot. trauma involves edema } \\
\text { and neuronal environment } \\
\text { that leads to apoptosis }\end{array}$ & $\begin{array}{l}\text { Gutierrez et al. (2001) and Run- } \\
\text { nerstam et al. (2001) }\end{array}$ \\
\hline & Rabbit & $\begin{array}{l}\text { Neurofilament redistribution } \\
\text { and beta-amyloid }\end{array}$ & $\begin{array}{l}\text { Effect of trauma on the } \\
\text { neuronal cytoskeleton }\end{array}$ & Hamberger et al. (2003) \\
\hline \multirow[t]{3}{*}{$\begin{array}{l}\text { Coronal plane rot. } \\
\text { acc. }\end{array}$} & $\begin{array}{l}\text { Miniature } \\
\text { swine }\end{array}$ & $\begin{array}{l}\text { Histology (NF), retraction } \\
\text { bulbs in the cerebral } \\
\text { hemispheres }\end{array}$ & & $\begin{array}{l}\text { Meaney et al. (1995) and Ross } \\
\text { et al. (1994) }\end{array}$ \\
\hline & Rat & $\begin{array}{l}\text { Silver staining, significant } \\
\text { damage to the brainstem }\end{array}$ & Development of test rig & Xiao-Sheng et al. (2000) \\
\hline & Rat & $\begin{array}{l}\text { Limited macroscopic brain } \\
\text { damage, no evidence of } \\
\text { axonal swellings }\end{array}$ & Transient unconsciousness & $\begin{array}{l}\text { Ellingson et al. (2005) and } \\
\text { Fijalkowski et al. (2007) }\end{array}$ \\
\hline $\begin{array}{l}\text { Transverse plane } \\
\text { rot. acc. }\end{array}$ & - & - & - & - \\
\hline $\begin{array}{l}\text { Oblique head rot. } \\
\text { acc. and contact }\end{array}$ & Sheep & $\begin{array}{l}\beta \text {-APP histology, contusions, } \\
\text { and axonal injury }\end{array}$ & $\begin{array}{l}\text { Axonal injury was related to } \\
\text { impact severity }\end{array}$ & Andersson, 2000 \\
\hline
\end{tabular}


To conclude, the number of models in which small animals are used and in which DAI is developed without large quantities of contusion type or hemorrhage injuries are limited. Therefore a new model in which the heads of rats are exposed to sagittal plane rotational accelerations resulting in graded levels of DAI has been developed and is presented here. This new model is intended for studies of inertial loading brain injuries to simulate forehead to hard structure impacts.

\section{MATERIALS AND METHODS}

The materials and methods used in this study are presented below. This paper is focused on the presentation of the test rig and its capabilities. The experimental data included were produced for studies with a special purpose and for this reason there is variability in the animal numbers used for different experiments. Additional information is available in Davidsson (2008) and in Risling et al. (2011).

\section{ANIMALS}

In total 110 male Sprague-Dawley rats were included in this study. Nineteen animals could not be included in the analysis due to various reasons. In five experiments the skull cap came loose during trauma. Eight additional animals died as a consequence of the anesthesia and six died either from the trauma or the anesthetics. The animals that died from the trauma likely suffered from acute brain stem injury which resulted in severe arrhythmia and respiratory arrest.

Out of the 110 animals, 91 male Sprague-Dawley rats weighing between 0.352 and $0.518 \mathrm{~kg}$ (with an average weight of $0.415 \mathrm{~kg}$ ) were either successfully exposed to rotational injury or served as sham exposed controls in this study (Table 2). In addition four animals were not operated on and served as normal controls. All animals were deeply anesthetized by a $2.4-\mathrm{ml} / \mathrm{kg}$ intra-abdominal injections of a mixture of $1 \mathrm{ml}$ Dormicum ${ }^{\circledR}(5 \mathrm{mg} / \mathrm{ml}$ Midazolam, Roche), $1 \mathrm{ml}$ Hypnorm ${ }^{\circledR}$ (Janssen), and $2 \mathrm{ml}$ of distilled water. Thereafter the subjects were given $0.2 \mathrm{ml} / \mathrm{kg}$ intra-muscular injections every $0.5 \mathrm{~h}$ until the surgery and trauma was carried out. The work was performed in accordance with the Swedish National Guidelines for Animal Experiments, which was approved by the Animal Care and Use Ethics Committee in either Umeå or Stockholm.

A midline incision was made through the skin and periosteum on the skull vault, and parts of the frontal, nose, and parietal bones were freed from adherent tissue. The exposed bone was treated with $15 \%$ phosphate acid for 3 min to clean and roughen the surfaces of the bone, thoroughly rinsed with tempered distilled water and dried for 3 min with an air drier providing air that was $37^{\circ} \mathrm{C}$ in the proximity of the skull bone. The exposed bone was then gently sanded, left to dry for at least an additional $10 \mathrm{~min}$ prior to gluing of an aluminum plate, denominated the skull cap (Figure 1) and shaped to match the contour of the exposed skull. The dental glue (Super-Bond C and B; Sun Medical Co., Shiga, Japan) was allowed to harden for a minimum of $15 \mathrm{~min}$. During the first $3 \mathrm{~min}$ the temperature was kept at $37^{\circ} \mathrm{C}$ after which it was adjusted to below but close to $37^{\circ} \mathrm{C}$.

\section{EXPERIMENTAL SETUP}

Prior to experiments, an attachment plate, that had a temperature close to $37^{\circ} \mathrm{C}$, was fastened by means of two screws to the skull cap previously glued to the rat skull bone (Figure 1). Then

Table 2 | Number of animals included in the various groups in this study (the numbers in the boxes refer to the total number of animals included in the group whereas the numbers in brackets refer to the number of sham exposed animals).

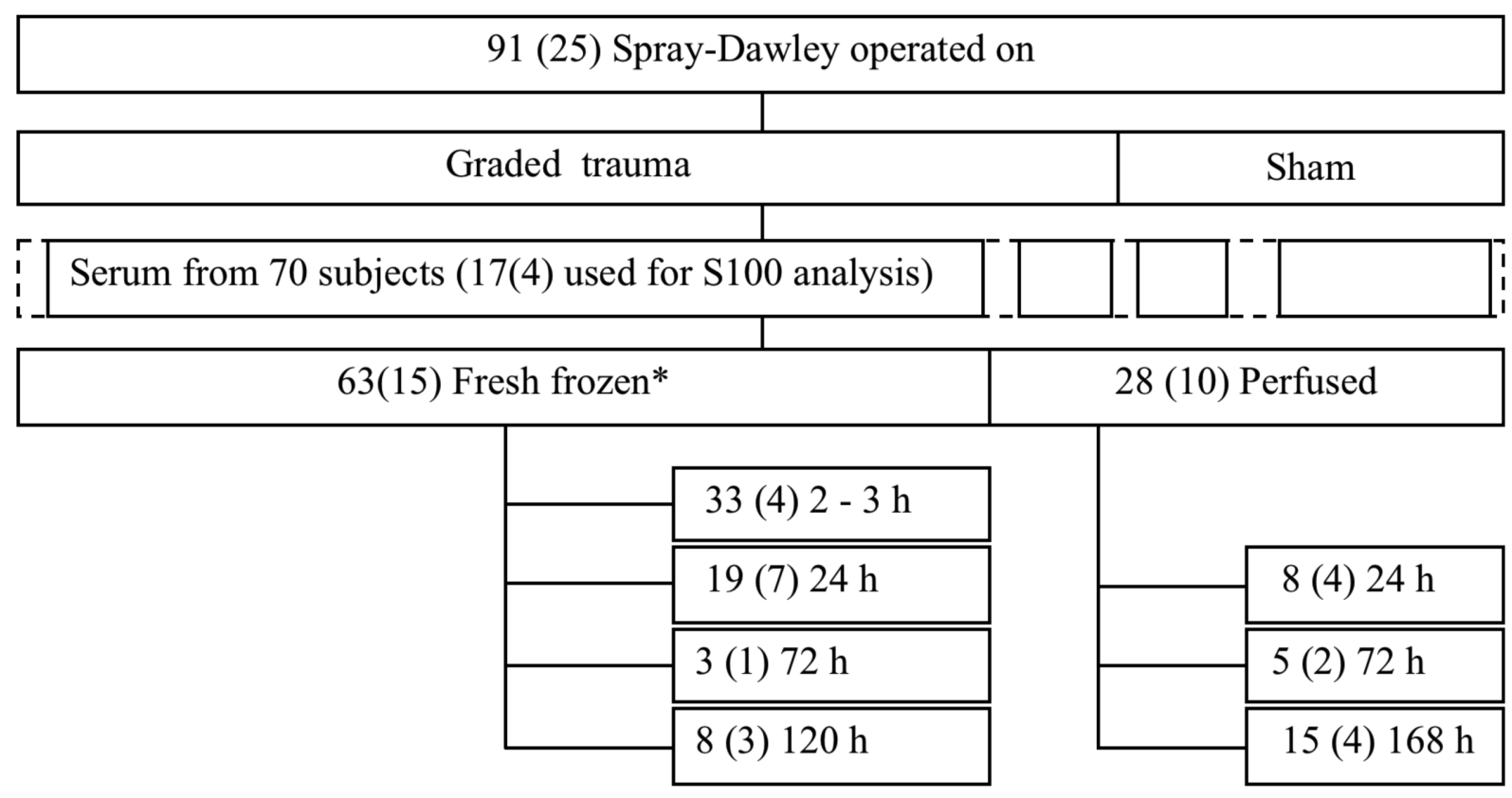

*63 animals used for $\beta$-APP analysis of which 31 was used for COX2 analysis. 




FIGURE 1 | Diagrams. Top row; skull cap and attachment plate and oblique view of test device (crossbeam removed for visibility). Bottom row; oblique view of test device and side view of the head with central nervous system schematically depicted (screws and accelerometer not depicted).

the attachment plate was inserted and secured to a rotating bar that can rotate freely around a horizontal axis. The resulting pretrauma position of the head was consistently slightly flexed and the brain center of gravity located about $6.5 \mathrm{~mm}$ above the center of rotation. This is equivalent to a center of rotation located $1 \mathrm{~mm}$ below the head base and $5 \mathrm{~mm}$ forward of the front of the foramina magnum.

Twenty-five animals served as sham exposed controls, thus 66 animals were traumatized. During trauma a weight, denominated the striker (brass, diameter $6.3 \mathrm{~mm}$, weight $0.010 \mathrm{~kg}$ ), was accelerated in a specially designed air driven accelerator (CNCProcess AB, Hova, Sweden) and was made to hit a rubber block (Polyurethane, Shore A 60, $10 \mathrm{~mm}$ thick, $13 \mathrm{~mm}$ wide, $10 \mathrm{~mm}$ high) that was glued onto a striker target (SS4212, aluminum plate $6 \mathrm{~mm}$ thick and $15 \mathrm{~mm}$ wide). The impulse produced subjected the rotating bar and the animal head to a short sagittal plane rearward rotational acceleration. The striker hit the rubber block at a height of $57 \mathrm{~mm}$ from the center of rotation of the rotating bar. This acceleration phase was followed by a rearward rotation at near constant velocity. Finally, the striker target made contact with the crossbeam (steel, $16 \mathrm{~mm}$ square profile) which was covered with high density Tempur foam $(20 \mathrm{~mm}$ thick and $20 \mathrm{~mm}$ high). The trauma represents a forehead impact to the steering wheel in a frontal car collision or to the ground in a fall accident. The rotational acceleration magnitude was selected by modifying the striker speed which was varied by means of modifying the air pressure in a specially designed air driven accelerator.

After trauma the attachment plate was removed, the skin was made to cover the skull cap and 8-10 sutures closed the incision.

\section{INSTRUMENTATION, DATA ACQUISITION, AND ANALYSIS}

An Endeveco Isotron 2255B-01 piezoelectric accelerometer, with integrated electronics and resonance frequency above $300 \mathrm{kHz}$, was mounted on the rotating bar at a radius of $36.5 \mathrm{~mm}$ from the center of rotation and connected to an Endeveco 4416B signal conditioner. The signal conditioner gain was set at one with an upper frequency cut off at $40 \mathrm{kHz}(-3 \mathrm{~dB})$ which served as antialiasing filter. The analog signal was digitized and captured by means of a National Instrument DAQ Card 6062 at $200 \mathrm{kHz}$. Thereafter the obtained rotational acceleration data was filtered using SAE J211 CFC3000 (5000 Hz).

A chronograph (SKAN PRO1 Series 3) was used to capture the velocity of the striker.

In four experiments, side views of the head trauma were recorded by a Redlake video at $20000 \mathrm{f} / \mathrm{s}$ with a resolution of $120 \times 68$ pixels. Oblique frontal views of the trauma were also recorded to monitor the rigidity of the head-to-test rig attachment.

Angular velocity and displacement were numerically integrated from the unfiltered acceleration data and compared with the high speed data for accuracy.

\section{DISSECTION, IMMUNOHISTOLOGY, IN SITU HYBRIDIZATION, AND SERUM ANALYSIS}

Post-trauma survival times were varied from $2 \mathrm{~h}$ to 7 days. The short survival times were preferred to assess the axonal injury as soon as possible after trauma to limit detection of secondary effects associated with the trauma or preparation of the animal; the longer survival time was required to detect contusion injuries and degenerative axons.

In case serum was to be collected prior to the sacrifice, the animals were anesthetized for at least $2 \mathrm{~h}$. Thereafter $5-7 \mathrm{ml}$ of blood were retrieved from the right ventricle of the heart with a Safety-Lock needle and SST II blood collection system containers (BD Vacutainer ${ }^{\circledR}$ ). The blood containers were filled and treated according to the instructions provided with the system. Serum was frozen immediately after centrifugation and stored at $-20^{\circ} \mathrm{C}$ until the analysis. In case no serum was to be collected, the animals were anesthetized for $15 \mathrm{~min}$ prior to being sacrificed.

After possible serum collection, 63 of the animals were killed, the brains were removed and split into three units at $0.6 \mathrm{~mm}$ and -3.0 relative to the bregma. The units were fresh frozen on dry ice. The three units are referred to as frontal, middle, and occipital. In total 28 animals were subjected to transcardial perfusion with phosphate buffered saline containing heparin and then fixed with formaldehyde in PBS after which the brain was subsequently removed. The brains were thereafter immersed in buffered formalin for at least a day and transferred for cold storage in sodium azide and sucrose solution in PBS.

Coronal $14 \mu \mathrm{m}$ cryostat sections from the three regions of the 63 fresh frozen encephalon sections were cut and thawed onto chromealum-gelatine treated slides. For a few animals also the brain stem was sectioned and thawed on slides. The frontal sections were from 1.5 to $0.5 \mathrm{~mm}$ relative to the bregma, the middle sections from 0 to $-1.0 \mathrm{~mm}$ relative to the bregma and occipital sections from 3.0 to $6.0 \mathrm{~mm}$ relative to the bregma. The sections were soaked in $0.01 \mathrm{M} \mathrm{PBS}$ buffer for $10 \mathrm{~min}$ and incubated over night in a humid chamber at $4^{\circ} \mathrm{C}$ with different antibodies. For detection of injured axons, sections were incubated with rabbit polyclonal $\beta$-APP antibodies (51-2700, Invitrogen, Corporation, CA, USA; dilution $1: 100)$. For studies of other brain injuries, sections were incubated with mouse monoclonal antibodies against blood brain barrier (BBB; SM-71, Sternberger Monoclonals Inc., Baltimore, 
MD, USA; dilution 1:200) and goat polyclonal antibodies against glial fibrillary acidic proteins (GFAP; 6170, Santa Cruz Biotechnology Inc., Santa Cruz, CA, USA; dilution 1:100). For studies of proliferation of macrophages, sections were incubated with mouse monoclonal anti-rat ED1 (MCA431R, AbD Serotec, Dusseldorf, Germany; dilution 1:1000). All antibodies were diluted in $0.01 \mathrm{M}$ PBS containing 5\% donkey serum, $5 \%$ Bovine serum albumin, $0.3 \%$ triton, and $0.1 \%$ sodium azide. The sections were then rinsed in $0.01 \mathrm{M}$ PMS and incubated for $45 \mathrm{~min}$ at $20^{\circ} \mathrm{C}$ with $0.01 \mathrm{M}$ PBS containing $0.1 \%$ sodium azide and $0.3 \%$ Triton and either Cy3-conjugated donkey anti-rabbit IgG, Cy3-conjugated donkey antimouse (Jackson Immuno Research Inc., PA, USA; diluted 1:500), Cy2-conjugated donkey anti-rabbit IgG, or Cy2conjugated donkey antigoat IgG (Jackson; diluted 1:500). After the sections were rinsed in PBS, they were mounted in a mixture of glycerol and PBS and cover slipped.

Similarly, perfused tissue was processed for detection of damage to neuronal processes with FD Neurosilver ${ }^{\mathrm{TM}}$ Kit I (PK301, FD Neurotechnologies Inc., Baltimore, MD, USA) according to the provided instructions.

In addition, sections from fresh frozen tissue from the frontal regions of nine animals that had been subjected to maximum rotational accelerations between 0.84 to $1.92 \mathrm{rad} / \mathrm{s}^{2}$, were fixated in ice cold $100 \%$ acetone for $10 \mathrm{~min}$ and rinsed in PBS buffer. Thereafter, they were incubated with the same $\beta$-APP antibody as above but this was diluted 1:100 in 0.01 M PBS buffer containing $5 \%$ BSA, $0.3 \%$ Triton and 5\% normal horse serum. Sections were incubated over night at $4^{\circ} \mathrm{C}$, rinsed in $0.01 \mathrm{M} \mathrm{PBS}$ buffer and then incubated with biotinylated anti-rabbit IgG (BA-1000, Vector Laboratories Inc., CA, USA) followed by biotinylated enzyme complex (VECTASTAIN Elite Kit, PK-6100, Vector Laboratories). Finally, the binding of the APP antibody was localized by incubation with DAB substrate (Vector, SK-4100). The sections were dehydrated and coverslipped with Entellan and examined using the $40 \times$ lens in a Nikon E600 microscope. Images were captured with a Nikon Digital Sight DS-U1 (5 megapixel) camera, controlled with Nikon EclipseNet software. A total of $279 \mathrm{APP}$-positive profiles at the border between the corpus callosum and the subcortical white matter were identified and outlined at a final magnification of $1240 \times$ with the polygonal marker. The area was recorded for each of these profiles. The results were analyzed with Graphpad Prism ${ }^{\circledR} 5$ software for Macintosh using Kruskal-Wallis one-way ANOVA and Dunn's multiple comparison test.
Cyclooxygenase 2 (COX2) is an enzyme responsible for formation of important biological mediators. It has been reported to be abundant in cells at sites of inflammation and has been suggested to correlate with cell death. For this analysis coronal sections from 31 animals were cut in an RNase free environment and thawed onto SuperFrost Plus ${ }^{\circledR}$ (MENZEL-GLÄSER, Germany) objectslides and stored in sealed boxes at $-70^{\circ} \mathrm{C}$ until used. Thereafter, a 48-mer Synthetic oligonucleotide COX2 probe was synthesized (CyberGene AB, Huddinge, Sweden). The probe was labeled at the $3^{\prime}$-end with deoxyadeno-sine-alpha-(thio)triphosphate, $(\alpha-$ 33P) $-3000 \mathrm{Ci} / \mathrm{mmol}, 10 \mathrm{mCi} / \mathrm{ml}$ (PerkinElmer, MA, USA) by using terminal deoxynucleotidyl-transferase (Fermentas AB, Sweden) and hybridized to the sections for $16-18 \mathrm{~h}$ at $42^{\circ} \mathrm{C}$. The sections were first rinsed in SSC and then in distilled water. Subsequently, dehydration in ethanol was performed. The sections were then counterstained with cresyl violet and mounted.

In the main part of the histological analysis brain sections from each of the three regions in the brain, frontal, middle, and occipital, were chosen arbitrarily and antibody reactivity was assessed with a NIKON E600 microscope equipped with a confocal $\mathrm{C} 1$ unit or using dark field microscopy. For $\beta$-APP and COX2, the analysis also included scoring according to a grading scheme (Table 3 ). In addition, sections from the brain stem were prepared and antibody reactivity assessed.

Two of the S100 proteins, S100A1B, and S100BB, found in the central nervous system glial cells, are known to be released into body fluids following trauma (Ingebrigtsen et al., 2000). In the current study multiple serum S100BB levels and the total S100B (S100A1B and S100B) levels were assessed using serum from 17 animals using two immunoassays (S100 EIA and S100B EIA, Can Ag Diagnostics AB, Gothenburg, Sweden).

\section{RESULTS}

\section{PERFORMANCE OF THE NEW TEST DEVICE}

The skull cap was larger in the first 17 experiments and had a size similar to the denuded nasal bone. As a consequence of this close match in size, the location of the skull cap relative to the head varied slightly in the first 17 experiments since the nasal shape of the animal varied. As a consequence, the center of rotation varied slightly in the first few experiments than in the rest of the series. No difference in histological outcome between the first 17 animals and the remaining animals could, however, be detected and the results obtained were included in the analysis.

Table 3 | Grading of $\beta$-APP and COX2.

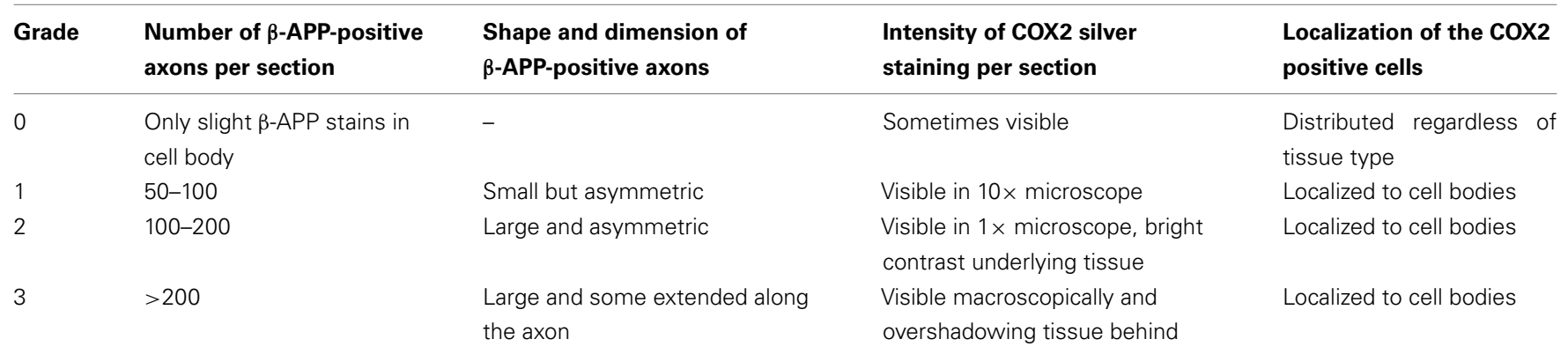




\section{ROTATIONAL AND LINEAR ACCELERATION AND VELOCITY CHANGE}

The charge pressure in the accelerator influenced the striker target speed which in turn influenced the rotational acceleration of the rotating bar and animal head unit. The duration of this angular acceleration was about $0.4 \mathrm{~ms}$ and was independent of striker velocity. As a consequence, the resulting angular velocity correlated closely with the peak angular acceleration $\left(r^{2}=0.86\right)$. After $10^{\circ}$ of head rotation, the striker target made contact with the foam covered crossbeam and the rotating bar and attached head came to a halt after about $2 \mathrm{~ms}$ at an angle of $20^{\circ}-30^{\circ}$ (Figure 2). Maximum deceleration was approximately $25 \%$ of initial peak acceleration (Figure 2). Thereafter the bar and the animal head slowly rotated forward to the position it had prior to trauma.

In this study the pressure in the accelerator was varied between 8 to 30 bar which resulted in striker velocities between 33 to $63 \mathrm{~m} / \mathrm{s}$ and maximum rotational accelerations between 0.3 to $2.1 \mathrm{rad} / \mathrm{s}^{2}$ (Figure 2).

In the experiments, the center of rotation was not perfectly in the center of gravity of the brain; hence the brain was exposed to a combination of rotational and linear accelerations. As an example, the maximum linear acceleration in the brain center of gravity ranged from 3600 to $7400 \mathrm{~m} / \mathrm{s}^{2}$ for the three experiments included in Figure 2.

Images of the rotating bar during trauma were recording using a high speed video for the purpose of evaluating the recorded linear acceleration and subsequent calculation of the rotational acceleration. The time from start to stop of the angulations of the head, obtained from video analysis, matched that of the accelerometer data. Resulting peak angulations of the rotating bar during the trauma, as estimated by double integration of the rotational acceleration, were also consistently about $20^{\circ}$ and matched those of the video data (Figure 2).

\section{DIFFUSE AXONAL INJURY}

Bands of $\beta$-APP-positive axons, i.e., axons with reduced axoplasmic transport and accumulation of $\beta$-APP, were seen in frontal sections and in the borders between the cortex and the corpus callosum (also forceps minor) in nearly all animals exposed to head rotational trauma of $1.0 \mathrm{Mrad} / \mathrm{s}^{2}$ or above (Figures 3 and 8). No $\beta$-APP reactivity was found in the sham exposed animals (Figure 3).

These bands were present independent of survival times, however the number of $\beta$-APP-positive axons appeared to increase when the survival time was $24 \mathrm{~h}$ compared with 3 and $2 \mathrm{~h}$. In animals that were exposed to rotational trauma at $1.3 \mathrm{Mrad} / \mathrm{s}^{2}$ or higher, bands of $\beta$-APP-positive axons were also seen in these borders in the middle and rear sections. This observation was not as consistent as for the frontal sections; animals that were allowed to survive for 5 days exhibited less positive axons in the middle and rear sections. Most animals displayed similar numbers of and intensity of $\beta$-APP-positive axons on the right and left hemispheres. The extensions of the $\beta$-APP-positive axons in the lateral direction varied from one animal to another.

For approximately $40 \%$ of the animals in which $\beta$-APP was found in the borders between the cortex and the corpus callosum,
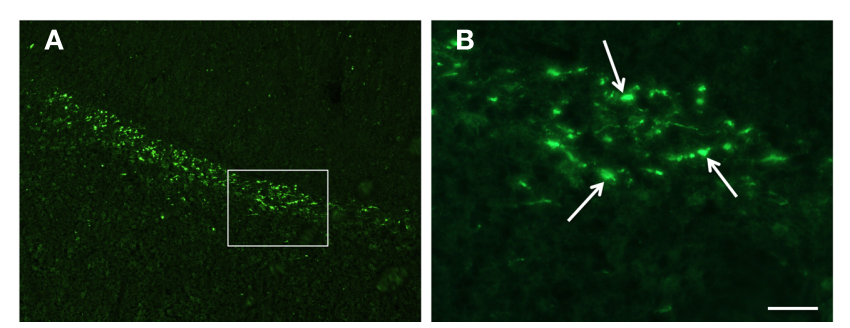

FIGURE 3 | Confocal images of $\beta$-APP stained tissue, coronal plane, frontal sections. In (A) a low magnification image shows the border between the corpus callosum (lower part) and subcortical white matter. A larger number of $\beta$-APP-positive profiles are visible at the border between the corpus callosum and the subcortical white matter, $24 \mathrm{~h}$ after high acceleration trauma. The box in (A) indicates the area that is shown in higher magnification in (B), in which $\beta$-APP-positive profiles have been indicated by arrows (scale bar $=25 \mu \mathrm{m}$ ).
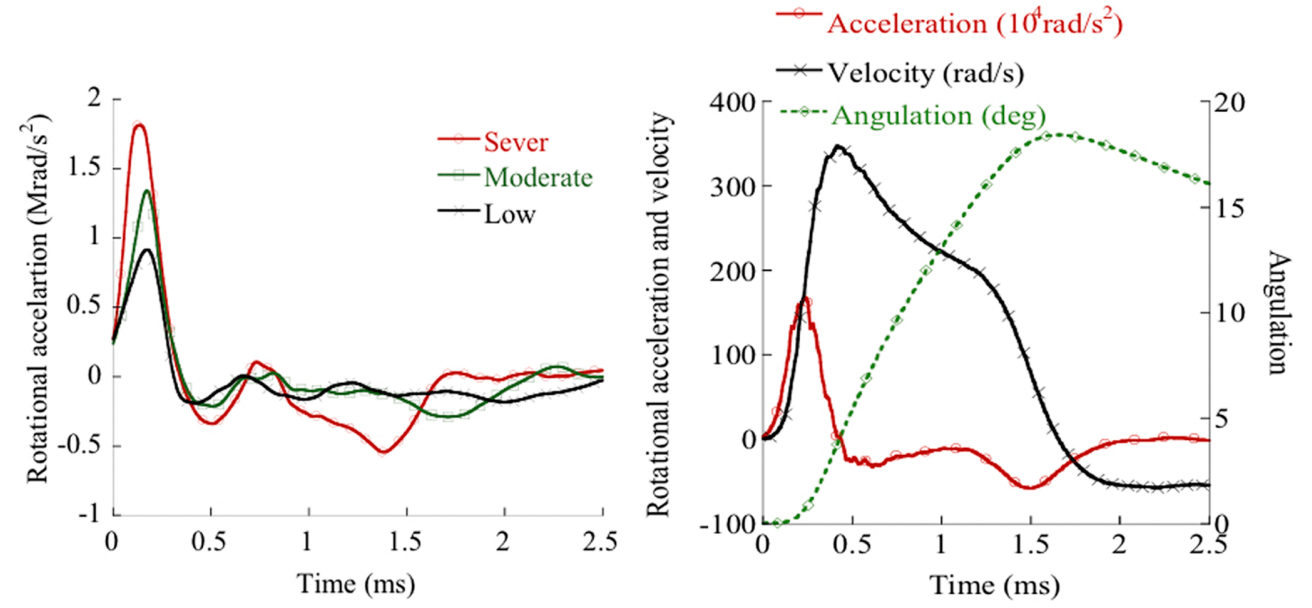

FIGURE 2 | Left: Rotating bar accelerations obtained in experiments that exhibited widespread, slight or no $\beta$-APP reactivity. Right: Rotational acceleration and integrated rotational acceleration (velocity). 
$\beta$-APP was also found in the thalamus, on the lower edges of the corpus callosum, in the corpus callosum, the caudate putamen and the commissure. In the middle brain sections, small diameter $\beta$-APP-positive axons were found in animals in the hippocampus region and in the vicinity of the lateral ventricle.

Seven brain stems were dissected and stained for $\beta$-APP analysis. Widespread areas with $\beta$-APP-positive axons were observed (Figure 4). As compared to the bands seen in the upper border of the corpus callosum, the numbers of positive axons were fewer. OBS! The highest concentration was found in the pyramid tracts.

In nine animals $\beta$-APP-positive profiles were analyzed with regard to size (area). No significant difference $(p<0.05)$ in size of the profiles could be detected, using the Kruskal-Wallis and Dunn's multiple comparison test. A straight line fitted to the mean values for the analyzed animals had a slope that did not significantly deviate from zero $\left(r^{2}=0.0008\right)$, suggesting that above the threshold for injury there is not a linear relationship between acceleration and mean size of the $\beta$-APP-positive profiles (Figure 5). Thus, increased acceleration does not seem to increase the size of the profiles.


FIGURE 4 | Fluorescence micrographs of sagittal plane sections from the pyramidal tract in brain stem incubated with $\beta$-APP (scale bar $=\mathbf{5 0} \boldsymbol{\mu} \mathbf{m}$; magnification in $(A)=(B))$. Twenty-four hours after high acceleration trauma a number of $\beta$-APP-positive axons are present (arrows).

Mean size of positive profiles

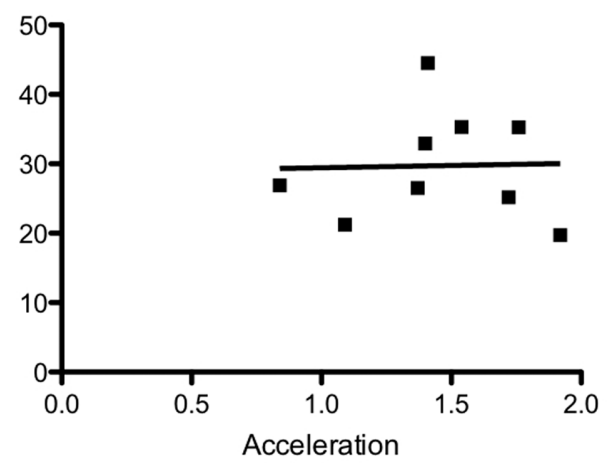

FIGURE 5 | Relationship between maximum acceleration and mean size of $\beta$-APP-positive profiles at the border between corpus callosum and subcortical white matter, using the $A B C$ method and image analysis, in nine animals $\mathbf{2 4} \mathbf{~}$ after trauma. The mean size $\left(\mu \mathrm{m}^{2}\right)$ of the analyzed profiles has been plotted against the acceleration. No correlation between size and acceleration was found.
The acceleration trauma also resulted in axonal FD silver labeling in the pyramid tracts in the brainstem (Figure 6). This was detected 7 days after severe trauma. It is most likely that silver labeled axons were present in other locations of the brain as well and perhaps at slightly shorter survival times. The silver labeling has not been analyzed in detail at present.

For the average levels of $\beta$-APP-positive axons, as determined according to the grading scheme previously presented (Table 3 ), a clear dose-response pattern can be observed; for $\beta$-APP-positive axons the increase starts at $1.1 \mathrm{Mrad} / \mathrm{s}^{2}$ (Figure 9).

\section{INFLAMMATORY RESPONSE}

An intense induction of COX2 mRNA following trauma was observed in the dentate gyrus within $3 \mathrm{~h}$ after trauma (Figure 7). A less intensive induction could be detected in some regions of the cortex and the basilar region. This was in contrast to what could be observed in the sham operated animals in which few or no cells were labeled. In the frontal sections, the COX2 was found mainly in the cingulated cortex and in the lateral regions of the cortex. In the middle sections, reactivity was found in the dentate gyrus, putamen/hippocampus region, and lateral regions of the cortex. In the occipital sections mainly the lateral regions of the cortex exhibited COX2 positive cells. The average levels of COX2 intensity, as subjectively determined according to the grading scheme

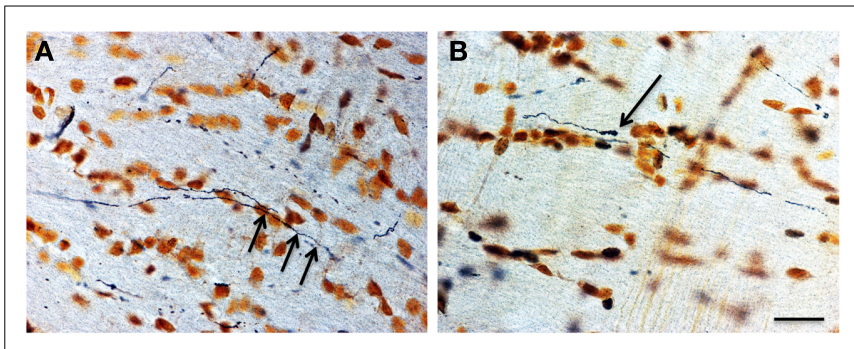

FIGURE 6 | Sections from the pyramidal tract. The micrographs show degenerating axons [(A) arrows] in the pyramidal tract 5 days after high level trauma, as revealed with the FD silver method. The axon in (B) had the appearance of an endbulb (scale bar $=25 \mu \mathrm{m}$; magnification in $A=B$ ).
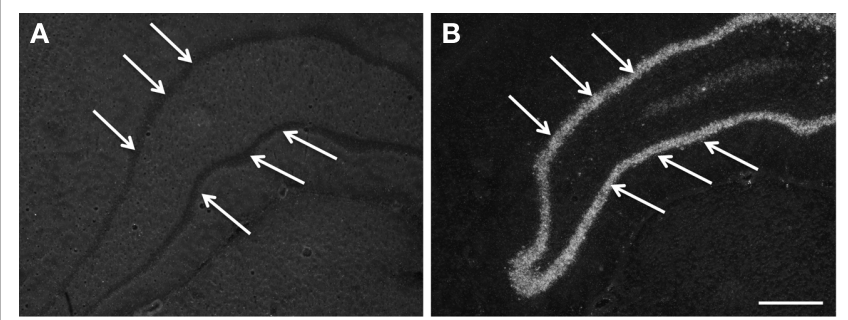

FIGURE 7 | Images, captured in darkfield microscopy, of coronal plane occipital sections of the dentate gyrus (arrows) after in situ hybridization for COX2 mRNA. (A) is a section from an animal exposed to a $0.7-\mathrm{Mrad} / \mathrm{s}^{2}$ rotational acceleration, whereas $(\mathbf{B})$ shows a section from an animal exposed to a severe rotational trauma (scale bar $=500 \mu \mathrm{m} ; A=B$ ). Note the absence of a signal in (A) and the intense signal in the dentate gyrus in (B). 




FIGURE 8 | Schematic graphs showing the middle coronal section of the rat brain. In (A) the stars indicate the localization of $\beta$-APP-positive axons. Note the distribution of positive profiles in corpus callosum, hippocampus, and thalamus. In (B) stars indicate the localization of cells with high expression of of COX2 mRNA. Note the high expression in dentate gyrus, cingulate cortex, as well as lateral and basal cortex.

previously presented (Table 3), increase with rotational acceleration (Figure 9). A clear dose-response pattern can be observed; for COX2 intensity the increase starts at around $0.9 \mathrm{Mrad} / \mathrm{s}^{2}$.

\section{OTHER INJURIES}

During sacrifice, hemorrhages were visible in the foramen magnum region; subdural and subarachnoid hemorrhages were observed on the superior cortex surface in slightly less than half of the exposed animals. A few animals suffered from hemorrhages in the vicinity of the olfactory bulb.

No invasions of ED1 positive macrophages or BBB changes could be observed. Macrophages were only very sparsely observed in the basal region of the brain and in a limited number of animals that had been exposed to high rotational acceleration.

Two repeated analyses of the total S100B levels revealed an increase when the maximum head acceleration was above $0.8 \mathrm{Mrad} / \mathrm{s}^{2}$ (Figure 9) whereas three repeated analyses of S100BB serum levels showed an increase when the acceleration was above $0.9 \mathrm{Mrad} / \mathrm{s}^{2}$.

\section{DISCUSSION}

The experimental data reveal that the new model produces graded injury. $\beta$-APP-positive axons and COX2 positive neurons were observed in the brain tissue and an increase of S100B in serum was detected when the rearward rotational acceleration was above $1.0 \mathrm{Mrad} / \mathrm{s}^{2}$ and the pulse duration was $0.4 \mathrm{~ms}$ (Figure 9). Increasing the trauma level resulted in prominent levels of $\beta$-APP, COX2, and S100B (Figure 9).

\section{DIFFUSE AXONAL INJURY}

Loads to an axon may cause an immediate axotomy, where the axons and myelin are simultaneously and directly damaged at the site of lesion, or a delayed disruptive injury to the axons. The latter is the most common type of injury to axons in animal models (Maxwell et al., 1993) and is commonly associated with axonal swelling whereas the former is associated with axonal bulbs. These swellings are a consequence of a sequence of events that includes perturbation to the axolemma, formation of nodal blebs, changes to the neurofilament structures and separation of the axolemma from the myelin sheath. Swelling occurs when the axonal transport system is affected which leads to the accumulation of transport material such as proteins and enzymes along the axons. The sequence may end with axonal disconnection and formation of axonal bulbs. Delayed or secondary axotomy is an important feature of TBI and DAI. This axonal injury is now known to be a complex array of responses to injuries of varying severity. In some cases, only the axonal cytoskeleton is injured and in other cases the adjacent axolemma show alterations in permeability, which may contribute to the disconnection of the affected axons. The timetable for such events is varying but can be assumed to continue for days (Farkas and Povlishock, 2007). We have not established the timetable for secondary axotomy in the present experiments. The presence of $\beta$-APP-positive profiles is assumed to be a good indicator of disrupted axonal transport, whereas the silver staining represents a marker for disconnected axons following the secondary axotomy.
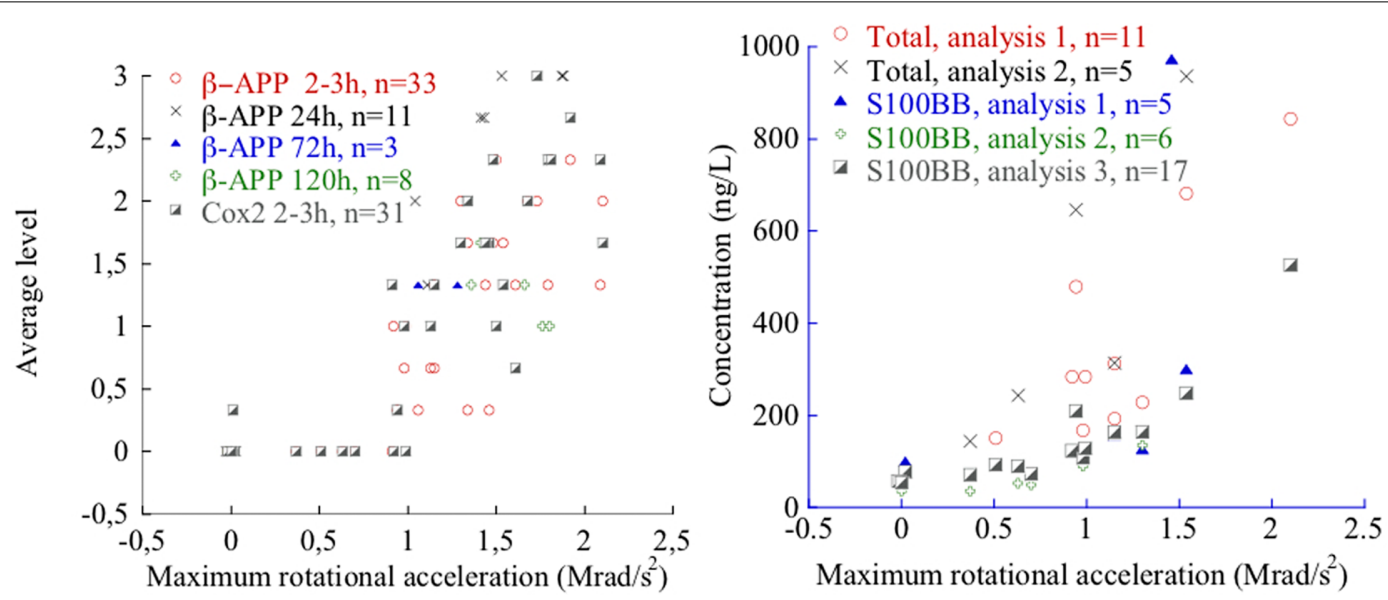

FIGURE 9 | $\beta$-APP and COX2 levels (left) and total S100B and S100BB levels after 2-3 $\mathrm{h}$ survival time (right) as a function of maximum rotational acceleration. Note that in the left plot, 12 sham exposed controls are included that all exhibited zero level $\beta$-APP and three sham exposed controls are included that all exhibited zero level of COX2. For one animal extremely high total S100B and S100 BB values were measured and are not depicted for clarity. 
In this study mainly three types of $\beta$-APP accumulations were present. These were used to assess risk of axonal injuries (AI) in the brain tissue following rotational trauma. In coronal sections dot like $\beta$-APP spots were found on the border between the corpus callosum and the cortex. Furthermore, axons that appeared swollen could be found in the corpus callosum (Figure 3). The dot like appearance was evidently a result of the fact that most of the effected axons were cut (Figure 3). In sagittal sections of the brain stem, axons with swollen appearance were found in abundance (Figure 4). There is no doubt that the new trauma model produces AI. Numerous studies have shown that $\beta$-APP is produced in neurons. The $\beta$-APP is transported distally in the axon and following an AI it accumulates at the site of injury (Gentleman et al., 1993; Blumbergs et al., 1994). However, due to the fact that most sections studied were coronal, the $\beta$-APP analysis alone is not enough to determine whether the new model mainly gave rise to secondary axotomy as a result of perturbation to the axolemma or also produced immediate axotomy.

To shed some light on this, tissue from a limited number of animals was stained using FD silver. The results confirmed the observations found after staining for $\beta$-APP and indicate that many of the injured axons degenerate after 7 days survival time. Future studies using stains to study filament changes (e.g., neurofilament and tubulin), as well as electron microscopy will be adopted to validate the method used to detect injury and to further assess the distribution of primary and secondary axotomy.

$\beta$-APP-positive axons were found in the edges of and inside the corpus callosum. The largest numbers were found in the superior edges of the frontal region of the corpus callosum. The distribution of injured axons may be an effect of the induced shearing and straining of the tissue during the trauma or/and may reflect an uneven distribution of axons as well as axons of different sizes. An overall density decline from the anterior to the posterior region of the corpus callosum was observed in a study by Riise and Pakkenberg (2011). These authors also found an inverse relationship between the distribution of large and small fibers.

The $\beta$-APP-positive profiles could be detected at a lower magnification in animals subjected to a higher acceleration injury. In fluorescence microscopy these profiles appeared larger. However, using $\mathrm{ABC}$ for $\beta$-APP and image analysis it was observed that there was no difference in mean size of $\beta$-APP-positive profiles between animals subjected to different levels of acceleration. This would imply that an intensity difference rather than a size difference can explain why the profiles can be detected by fluorescence microscopy at a lower magnification in animals that have sustained a higher acceleration. Unfortunately, we have no reliable method to measure the intensity in the fluorescence signal, but the possibility that a larger acceleration injury might induce a more drastic accumulation of $\beta$-APP that could lead to a higher concentration of the protein cannot be ignored.

The localization of the affected axons in this study partly resembles those commonly reported in the literature following DAI Grade I: characterized by microscopical AI mainly in the corpus callosum and the parasagittal white matter (Adams et al., 1989). The near absence of positive axons in the white matter of the cerebral hemispheres in this study may reflect species differences. Alternatively, they do not appear after the type of trauma used in this study. It has also been reported that DAI is associated with lesions in the brain stem and cerebellum (DAI Grade 3). Preliminary findings, including $\beta$-APP-positive axons, FD silver labeled axons, and GFAP changes in the upper brainstem region as studied in about 10 animals suggest that the model also produces DAI grade 3 .

Based on the histological findings in this study we are confident that the model used in this study produces DAI since distributed $\mathrm{AI}$ are produced in representative regions of the brain and brainstem. Future studies will further categorize the extent of the axonal injury produced by this trauma model.

\section{INFLAMMATORY RESPONSE}

Cyclooxygenase converts arachidonic acid to prostaglandin $\mathrm{H} 2$, a precursor of the prostanoids, which is a subclass of eicosanoids that includes prostaglandins, thromboxane and prostacyclin. Different COX isoenzymes are known. COX1 is a constitutive enzyme in most mammalian cell types, whereas COX2 is an enzyme that can be induced in macrophages and other cells at sites of inflammation or injury. Our observation of an upregulation of COX2 mRNA in areas such as the dentate gyrus after rotational TBI is compatible with our previous observation that gene clusters relating to inflammation show a significantly changed expression in the hippocampus after this type of injury (Risling et al., 2011). Future behavioral studies tailored to study memory deficiencies and anxiety reactions, e.g., radial maze and elevated plus, will be adopted to assess the effects of the observed inflammation in the hippocampus region following rotational trauma.

\section{OTHER INJURIES}

Some previous studies classify brain injury according to the presence of neuropathological changes and the frequency and duration of unconsciousness following trauma (Ono et al., 1980; Gennarelli et al., 1982). In this study the severity of the concussion was not assessed and as such makes a direct comparison difficult.

Contusions have been present following rotational trauma in studies in the past. In this study tissue stained to study astrocyte changes, macrophage invasions, and permeability of BBB indicated that there were no contusions related to the trauma.

Preliminary results from a behavioral study, in which changes to the balance was assessed using a beam walk test, indicate that no major neck muscle injuries or inner ear injuries occurred during the rotational trauma (manuscript in preparation Risling et al., 2011).

Hemorrhages were observed in the foramen magnum region in a large proportion of the animals after the very first few tests using this model. To reduce these injuries, head rearward rotation was limited to approximately $20^{\circ}$ during the trauma. Also, the mounting position of the skull cap had an effect on these injuries; when the head center of rotation was shifted forward there were more hemorrhages in the meninges in the brainstem region and also an increased number of deaths immediately after trauma. In a limited number of cases, hemorrhages were also found in the meninges surrounding the olfactory bulbs and on the top of the hemispheres. The incidence rate of hemorrhages did not appear to correlate to the head angle or the position of the center of rotation. Similarly, 
only an insignificant correlation between the extent of hemorrhages and rotational acceleration was observed: no hemorrhages were found in unexposed control animals, but they were observed in some of the animals when exposed to rotational trauma above $0.6 \mathrm{Mrad} / \mathrm{s}^{2}$.

Following moderate level of rotational head trauma we recorded elevated $\mathrm{S} 100 \mathrm{~B}$ and $\mathrm{S} 100 \mathrm{BB}$ levels in serum despite the absence of obvious BBB injuries. The trauma could result in minor BBB injuries, which was not detectable using traditional histology, responsible for the S100B leakage. Future studies using MRI may shed some light on this discrepancy.

In this study, we have shown that the developed model produces axonal injury as detected by $\beta$-APP-positive axons and a possible inflammatory response as we have seen an intense induction of COX2 stained nerve cells. In addition we have measured a steady increase in concentrations of S100B and S100BB in serum following rotational acceleration trauma. Since the three markers become apparent and abundant in a rather narrow trauma severity interval, it may be suggested that the three markers indicate one single type of brain tissue injury. This injury would most likely be injuries to the axons, but could also be a combination of $\mathrm{AI}$ and

\section{REFERENCES}

Abel, J. M., Gennarelli, T. A., and Segawa, H. (1978). "Incidence and severity of cerebral concussion in the rhesus monkey following sagittal plane angular acceleration," in Proceedings of the 22nd Stapp Car Crash Conference, Ann Arbor: MI, 780886, 35-53.

Adams, J. H., Doyle, D., and Ford, I. (1989). Diffuse axonal injury in head injury: definition, diagnosis and grading. Histopathology 15, 49-59.

Andersson, R. W. G. (2000). A Study on the Biomechanics of Axonal Injury. Thesis, University of Adelaide.

Blumbergs, P. C., Scott, G., Manavis, J., Wainwright, H., Simpson, D. A., and McLean, A. J. (1994). Staining of amyloid precursor protein to study axonal damage in mild head injury. Lancet 344, 1055-1056.

Davidsson, J. (2008). A New Model, Experiments and Injury Threshold for Sagittal Plane Rotational Induced Diffuse Brain Injuries. Report No. AP-SP51-0045, Generated in Project The 6th Frame Work EU-project APROSYS. Available at: http://www.aprosys.com/

Ellingson, B. M., Fijalkowski, R. J., Pintar, F. A., Yoganandan, N., and Gennarelli, T. A. (2005). New mechanism for inducing closed head injury in the rat. Biomed. Sci. Instrum. 41, 86-91. PMID: 15850087.

Farkas, O., and Povlishock, J. T. (2007). Cellular and subcellular change evoked by diffuse traumatic brain injury: a complex web of change extending far beyond focal damage. Prog. Brain Res. 161, 43-59.

Fijalkowski, R. J., Stemper, B. D., Pintar, F. A., Yoganandan, N., Crowe, M. J., and Gennarelli, T. A. (2007). New rat model for diffuse brain injury using coronal plane angular acceleration. J. Neurotrauma 24, 1387-1398.

Foda, M. A., and Marmarou, A. (1994). A new model of diffuse brain injury in rats. Part II: morphological characterization. J. Neurosurg. 80, 301-313.

Gennarelli, T. A., and Thibault, L. E. (1982). Biomechanics of subdural hematoma. J. Trauma 22, 680-686.

Gennarelli, T. A., Thibault, L. E., Adams, J. H., Graham, D. I., Thompson, C. J., and Marcincin, R. P. (1982). Diffuse axonal injury and traumatic coma in the primate. Ann. Neurol. 12, 564-574.

Gentleman, S. M., Nash, M. J., Sweeting, C. J., Graham, D. I., and Roberts, G. W. (1993). Beta-amyloid precursor protein (beta APP) as a marker for axonal injury after head injury. Neurosci. Lett. 160, 139-144.

Gutierrez, E., Huang, Y., Haglid, K., Bao, F., Hansson, H. A., Hamberger, A., and Viano, D. (2001). A new model for diffuse brain injury by rotational acceleration. J. Neurotrauma $18,247-257$.

Hamberger, A., Huang, Y. L., Zhu, H., Bao, F., Ding, M., Blennow, K., Olsson, A., Hansson, H. A., Viano, D., and Haglid, K. G. (2003). Redistribution of neurofilaments and accumulation of beta-amyloid protein

contusion injuries to the surface of the brain. However, as mentioned earlier, no macrophage invasion or BBB changes could be observed and COX2 stained nerve cells along the periphery of the brain were only observed sparsely in the basal regions of the brain. These observations indicate that the model produces AI with only minor contusion injuries.

\section{CONCLUSION}

We conclude that the developed animal model is capable of producing graded DAI with limited contusion injury. We also conclude that the model is inexpensive to use and produces a reproducible injury panorama. The animal model produces AI mainly in the corpus callosum, on the upper and lower boundaries of the corpus callosum, and in the brain stem, which commonly has been reported in studies of DAI.

\section{ACKNOWLEDGMENTS}

The study was funded by Karolinska Institutet, APROSYS 6th Frame Work, The Swedish Armed Forces and Vinnova, Sweden. We thank Mrs. Maria Angeria for excellent technical assistance and Viktoria Hammarstedt for editorial help.

after brain injury by rotational acceleration of the head. J. Neurotrauma 20, 169-178.

Hansson, H. A., Höjer, S., and Krave, U. (2003). "Increased neurogenesis and diffuse brain damage after rotational acceleration of the head," in The 21st Annual National Neurotrauma Society Symposium, Biloxi, MS.

Holbourn, A. H. (1943). Mechanics of head injuries. Lancet 2, 438-441.

Ingebrigtsen, T., Romner, B., MarupJensen, S., Dons, M., Lundqvist, C., Bellner, J., Alling, C., and Børgesen, S. E. (2000). The clinical value of serum S-100 protein measurements in minor head injury: a Scandinavian multicentre study. Brain Inj. 14, 1047-1055.

Kleiven, S. (2007). "Predictors for traumatic brain injuries evaluated through accident reconstructions," in Proceedings of the 51st Stapp Car Crash Conference, San Diego: CA, Vol. 51, 07S-27S.

Krave, U., Al-Olama, M., and Hansson, H. A. (2011). Rotational acceleration closed head flexion trauma generates more extensive diffuse brain injury than extension trauma. J. Neurotrauma 28, 57-70.

Krave, U., Höjer, S., and Hansson, H. A. (2005). Transient, powerful pressures are generated in the brain by a rotational acceleration impulse to the head. Eur. J. Neurosci. 21, 2876-2882.

Margulies, S. S., and Thibault, L. E. (1992). A proposed tolerance criterion for diffuse axonal injury in man. J. Biomech. 25, 917-923.
Margulies, S. S., Thibault, L. E., and Gennarelli, T. A. (1990). Physical model simulations of brain injury in the primate. J. Biomech. 23, 823-836.

Marmarou, A., Foda, M. A., van den Brink, W., Campbell, J., Kita, H., Demetriadou, K., Maxwell, W. L., Watt, C., Graham, D. I., and Gennarelli, T. A. (1994). A new model of diffuse brain injury in rats. Part I: pathophysiology and biomechanics. J. Neurosurg. 80, 291-300.

Maxwell, W. L., Watt, C., Graham, D. I., and Gennarelli, T. A. (1993). Ultrastructural evidence of axonal shearing as a result of lateral acceleration of the head in non-human primates. Acta Neuropathol. 86, 136-144.

Meaney, D. F., Smith, D., Ross, D. T., and Gennarelli, T. A. (1993). "Diffuse axonal injury in the miniature pig: biomechanical development and injury threshold," in Crashworthiness and Occupant Protection in Transportation Systems, Eds J. D. Reid and K. H. Yang (NewYork: American Society of Mechanical Engineers), AMD-Vol. 169/BED-Vol. 25 (ASME), 169175.

Meaney, D. F., Smith, D. H., and Shreiber, D. I. (1995). Biomechanical analysis of experimental diffuse axonal injury. J. Neurotrauma 12, 689-694.

Melvin, J., Lighthall, W. J., and Ueno, K. (1993).Accidental Injury, Biomechanics and Prevention. New York: Springer-Verlag. 
Davidsson and Rising

Rotational TBI

Ommaya, A. K. (1984). "The head: kinematics and brain injury mechanisms," in The Biomechanics and Impact Trauma, eds B. Adman and A. Chapon (Amsterdam: Elsevier), 117-125.

Ono, K., Kikuchi, A., Nakamura, M., Kobayashi, H., and Nakamura, N. (1980). "Human head tolerance to sagittal impact reliable estimation deduced from experimental head injury using subhuman primates and human cadaver skulls," in Proceedings of the 24th STAPP Car Crash Conference, Troy: MI, 801303, 101-160.

Povlishock, J. T. (1992). Traumatically induced axonal injury: pathogenesis and pathobiological implications. Brain Pathol. 2, $1-12$.

Povlishock, J. T., and Jenkins, L. W. (1995). Are the pathobiological changes evoked by traumatic brain injury immediate and irreversible? Brain Pathol.5, 415-426.

Rinse, J., and Pakkenberg, B. (2011). Stereological estimation of the total number of myelinated callosal fibers in human subjects. J. Anat. 218, 277-284.

Risling, M., Planman, S., Angeria, M., Rostami, E., Bellander, Kirkegaard, M., Arborelius, U., and Davidsson, J. (2011). Mechanisms of blast induced brain injuries, experimenal studies in rats. Neuroimage 54S1, S89-S97.

Ross, D. T., Meany, D. F., Sabol, M. K., Smith, D. H., and Gennarelli, T. A. (1994). Distribution of forebrain diffuse axonal injury following inertial closed head injury in miniature swine. Exp. Neurol. 126, 291-299.

Runnerstam, M., Boo, F., Huang, Y., Chi, J., Gutierrez, E., Hamberger, A., Henson, H. A., Vino, D., and Haglid, K. (2001). A new model for diffuse brain injury by rotational acceleration: II. Effects on extracellular glutamate, intracranial pressure, and neuronal apoptosis. J. Neurotrauma 18, 259-273.

Smith, D. H., Chen, X. H., Xu, B. N., McIntosh, T. K., Gennarelli, T. A., and Meany, D. F. (1997). Characterization of diffuse axonal pathology and selective hippocampal damage following inertial brain trauma in the pig. J. Neuropathol. Exp. Neurol. 56, 822-834.

Smith, D. H., and Meany, D. F. (2000). Axonal damage in traymetic brain injury. Neuroscientist 6 , 483-495.

Strich, S. J. (1961). Shearing of nerve fibres as a cause of brain damage due to head injury. Lancet 2, 443.

Vino, D. C. (1997). Brain Injury Biomechanics in Closed-Head Impact. Ph.D. thesis, Karolinska Institute, Solna.

Wismans, J., Jansen, E., Beusenberg, M., and Bovendeerd, P. (2000). Injury Bio-Mechanics. Course Book w5-pp3-4.3, Eindhoven: Eindhoven Technical University.

Xiao-Sheng, H., Sheng-Yu, Y., Xians, Z., Chou, F., and Jian-ning, Z. (2000). Diffuse axonal injury due to lateral head rotation in a rat model. J. Neurosurg. 93, 626-633.

Chang, L., Yang, K. H., and King, A. I. (2004). A proposed new injury tolerance for mild traumatic brain injury. J. Biomech. Eng. 126, 226-236.

Conflict of Interest Statement: The authors declare that the research was conducted in the absence of any commercial or financial relationships that could be construed as a potential conflict of interest.

Received: 01 February 2011; paper pending published: 18 February 2011; accepted: 08 June 2011; published online: 17 June 2011.

Citation: Davidson $J$ and Rising $M$ (2011) A new model to produce sagital plane rotational induced diffuse axonal injuries. Front. Neur. 2:41. do: 10.3389/fneur.2011.00041

This article was submitted to Frontiers in Neurotrauma, a specialty of Frontiers in Neurology.

Copyright (C) 2011 Davidson and Risling. This is an open-access article subjest to a nonexclusive license between the authors and Frontiers Media SA, which permits use, distribution and reproducion in other forums, provided the original authors and source are credited and other Frontiers conditions are complied with.

www.frontiersin.org

June 2011 | Volume 2 | Article 41 | 11 